\title{
El ciclo de vida de un área turistica: su aplicacion a la Costa del Sol española y su medio ambiente
}

\author{
Pamela L. PÉREZ \\ Profesora de la Universidad Estatal de California \\ pamela.1.perez@csun.edu
}

Recibido: 21 de febrero del 2012

Enviado a evaluar: 24 de febrero del 2012

Aceptado: 27 de julio del 2012

\begin{abstract}
RESUMEN
El Ciclo de vida de un área turística, formulado por Richard W. Butler, tiene siete fases de evolución que se le aplican al destino turístico. En el concepto, se trata a esta localidad como si fuese un organismo viviente. Es decir, nace, florece; si padece de algún achaque, puede morir o de acuerdo con su realidad, renacer. A través de esta teoría también se puede estipular la sostenibilidad de un destino turístico, ya que, solamente se puede analizar la capacidad o múltiples capacidades de acogida dentro de su posición en el ciclo. En el caso de la Costa del Sol española, es la primera vez que se le aplica esta teoría y se determina que no encaja completamente dentro de una sola fase. Así, es necesario encajarlo dentro de su propio estadio, designado aquí, como el del "pre-estancamiento".

También, se concluye que en el presente, dada la distorsión entre su capacidad de acogida ambiental del agua, por el consumo, en exceso, que hacen los campos de golf de este bien, y la consecuente capacidad negativa de carga antropológica, es un destino turístico insostenible. Sin embargo, tiene la capacidad de superar esta situación, siempre y cuando, recicle sus recursos acuíferos para el riego de estos campos deportivos.
\end{abstract}

Palabras clave: Agua, Campos de Golf, Capacidad de acogida, Medio ambiente, Reciclaje, Sostenibilidad, Turismo.

\section{The tourist area life cycle: s application to the spanish Costa del Sol and its environment}

\begin{abstract}
The Theory of a Tourism Area Life Cycle, formulated by Richard W. Butler, has seven phases of evolution, which are applied to the tourism destination. In this concept, the location is treated as if it were a living organism. That is to say, it is born, it flourishes, if it develops an illness, it may die or in accordance with its particular reality it may be reborn.

This theory may also stipulate the sustainability of a tourism destination, as its carrying capacity, or multiple capacities, may only be analyzed within its position in the cycle. This is the first time the theory has been
\end{abstract}


applied to the Spanish Costa del Sol and it is determined, that this destination does not fit completely in any one phase. Therefore, it is necessary to create its own series, by designating it, as that of "pre-stagnation". Also, it is concluded, that, at present, given the distortion between its aquatic environmental carrying capacity, due to the consumption of this resource, by the golf courses and its consequential negative social carrying capacity, it is an unsustainable tourism destination. Though, it does have the capacity to overcome this situation, by recycling the water used for irrigating these courses.

Keywords: Water, Carrying Capacity, Environment, Recycling, Sustainability, Tourism.

\section{INTRODUCCIÓN}

El concepto del Ciclo de vida de un área turística (CVAT) se aproxima a este espacio recreativo como si se tratase de un organismo viviente. Es decir, esta localidad nace, vive, crece, pasa por sus achaques, y termina en el ocaso, si se ha descuidado demasiado en sus enfermedades. Si se encuentra una cura para sus males, el destino puede renacer y seguir floreciendo. Todo ese proceso se puede comparar con el fenómeno de la dialéctica, visto más particularmente, y aplicado a un espacio geográfico específico, en vez de a un cuerpo pensante. Es la vida misma. Se vive, lo que es la tesis. Se muere, lo que es la antitesis. Si el espacio termina en el declive o en el rejuvenecimiento, es la síntesis.

La teoría del CVAT se dio a conocer en 1.972 por Richard W. Butler y James Brougham en una conferencia del Travel Research Association canadiense. Al darse a conocer, en un principio, no causó gran sensación, en los estudios de turismo de aquel entonces. No fue hasta la década de los ochenta, cuando empezó a llamar la atención de los científicos en esta rama del saber. Así, y desde aquel entonces, se ha llegado a aplicar en regiones tan diversas como Hawai, la Costa Dorada de Australia, pasando por Venecia, y hasta Lancaster Pensilvania, tierra de los ammish. Lo que nunca se ha hecho, hasta el momento, es aplicarlo al destino turístico de la Costa del Sol española. Esto es precisamente lo que se pretende llevar a cabo en este estudio de investigación.

\section{EL ESPACIO REGIONAL DE LA COSTA DEL SOL}

La región de estudio de este análisis consiste en ocho subregiones, incluyendo el piedemonte costero. De este a oeste se comienza con Málaga, como capital de la provincia y localidad urbana más grande de la costa inmediata. Se sigue con la subregión de Churriana. Este pueblo cae bajo la jurisdicción legislativa de Málaga y sus campañas turísticas ecológicas o "verdes" son casi idénticas.

La siguiente localidad, camino al oeste, también bajo el auspicio legislativo de la capital provincial, además, donde se ha explotado y se sigue explotando sus sustanciales recursos recreativos, es Torremolinos. Aunque, en la actualidad, no ofrece un campo de golf, como atracción deportiva. Esta situación se puede clasificar como laguna turística en el espacio de la también llamada, Costa del Golf, debido a sus más de 82 
campos construidos para la persecución de este entretenimiento. Históricamente, sin embargo, Torremolinos, sí contaba con una instalación para este deporte.

También cabe destacar, dentro de la evolución de esta industria, en Torremolinos, que irónicamente, el nacimiento del turismo, en esta localidad, no tuvo nada que ver con su proximidad geográfica al aeropuerto, como suele ser el caso con la mayoría de estos destinos españoles. Sin embargo, esta coyuntura fue básica para su desarrollo posterior como centro turístico.

El próximo pueblo, siguiendo la misma dirección occidental es Benalmádena.

En Benalmádena:

"However, as in Torremolinos, there is considerable variety within the municipal area itself, reflecting the operation of land speculation and landownership patterns at various phases of the area's development. In the mid-1950's to the mid-1960's such activity was concentrated on the flatter areas in the east of the municipio, with the fragmentation of some large units of ownership into smaller parcels for direct sale to future occupants of villas and chalets. At the same time, in the Benalmádena Costa area, a number of small land units were being consolidated by a property company with a view to developing a large urbanización. From the mid-1960's to the early 1970's actual development proceded much more rapidly and was located more in the center and west of the municipal coastal area."1

Este municipio contrasta, por ejemplo, con los municipios de Mijas y Marbella, durante una época, en que la mayoría de la propiedad desarrollada con fines turísticos estaba en manos de los españoles. Originalmente estas propiedades eran cortijos, lo que daba al espacio de Benalmádena una caractrística fragmentada y dispersa. Este tipo de desarrollo de turismo residencial tiende a producir el efecto de viviendas de aislamiento, las unas de las otras.

Siguiendo con el recorrido occidental, se encentra Fuengirola. En esta localidad también predominan los hoteles de tres estrellas y turismo de masas, como se da en Torremolinos y Benalmádena. La diferencia entre ellos radica en el componente sociológico de la industria ya que predominan los visitantes en familias. Notablemente, la mayoría de las propiedades que se pueden adquirir en Fuengirola son del tipo de pequeños chalets, apartamentos o, más recientemente las multipropiedades en tiempo compartido.

Hace falta hacer una pausa en el municipio de Mijas, localizado al oeste de Fuengirola. Este lugar destacaba por ser, históricamente, un asentamiento dedicado a la agricultura, que paulatinamente se fue convirtiendo en urbanización turística. Aquí se sitúa el campo de golf más grande de España, que incluye hotel, balneario y varios tipos de residencias, en forma de pisos de lujo, chalets adosados o las más caras, villas semiadosadas. Un hecho significativo y a la vez simbólico, es que el

1 Barke, M. y L.A. France (1996). “The costa del Sol”, en M. Barke et al. (eds.), Tourism in Spain: Critical Issues, CAB International, Wallingford, p. 280. 
complejo es propiedad de un grupo de inversionistas irlandeses. Este hecho coloca a Mijas en el segundo puesto en cuanto a la inversión extranjera, en los destinos turísticos de la Costa del Sol.

Siguiendo hacia el poniente, yace Marbella. Esta localidad destaca por dos razones dentro del entorno Costa Soleño. Primero, y dentro del encuadramiento de la inversión extranjera, aquí, la pauta ha favorecido este tipo de actividad económica. Esta situación se da, comparativamente, con la región y con España, en general, aunque ha habido participación, en su desarrollo, de señalados y conocidos individuos españoles. En segundo lugar, y en contraste con los municipios ya mencionados, también destaca por atraer a visitantes y residentes de más elevada categoría económica y social.

Más próxima a la posesión británica de Gibraltar que al aeropuerto de Málaga, se encuentra la localidad de Estepona. En el pasado, era un destino turístico más orientado al mercado nacional, y sólo recientemente ha encontrado un sitio dentro de esta industria, en el nivel internacional. Esto se ha conseguido mediante la inserción de su suelo en el mercado de turismo residencial, tan importante en el panorama turístico contemporáneo nacional. Esta transformación tomó lugar sobre todo después de la muerte de Franco, cuando se establecieron relaciones sociales, económicas y políticas entre la Nación Española y Gibraltar.

Se espera que este fenómeno urbanístico no se asemeje al desarrollo de Mijas, ya que existe una mayor reglamentación en cuanto a la planificación urbanística del litoral, en la actualidad, que la que había en el pasado. Sin embargo, en Gibraltar, las agencias de bienes inmuebles anuncian la venta de hermosos chalets en la costa de Estepona. Estos representan una adquisición a muy buen precio para el bolsillo inglés, por el tipo de cambio entre el euro y la generalmente poderosa libra esterlina.

Actualmente en España se apuesta por este tipo de turismo residencial que se da muy extensamente en Estepona. Aporta más dinero a la economía en comparación con el turismo barato de los hoteles de tres estrellas. Sin embargo, estas estructuras son las que más carga representan para el medio ambiente. De esta manera:

"El modelo de urbanizaciones de chalet con piscina y campo de golf lleva implícito el despilfarro del agua. La Costa del Sol es un monstruo insaciable.

La principal razón es que el agua no es únicamente un bien de primera necesidad, sino un elemento que otorga estatus social mediante el jardín, la piscina o el campo de golf. Estatus que se mide en metros cuadrados de espacio verde y eso implica el desmedido consumo del agua. Un síntoma es que la media de consumo por habitante es de cuatrocientos litros al día, muy por encima de la media española. El diagnóstico lo hace Javier de Luis, coordinador de Ecologistas en Acción en Marbella. ${ }^{2}$

2 Bejarano, José (2005). "El monstruo insaciable de la Costa del Sol”, La Vanguardia, España, 2 de agosto. 
Esta cita es aplicable al ayuntamiento de Estepona y a cualquier otro que se encuentre en el litoral de la España seca. Esta región se denomina como tal al recibir menos lluvia que la media nacional. Toda la costa tratada, en este estudio de investigación, se enmarca dentro de esta zona falta de precipitación, y por lo tanto, se encuentra, en total desacuerdo con sus necesidades extremas de consumo individual de la población, además de, sus usos colectivos del turismo.

En la puesta en escena de este estudio, se le aplica a la región, las fases de desarrollo pertinentes, del Ciclo de vida de un área turística. La conclusión de dicha teoría, en el caso de este análisis, refleja principalmente la distorsión de la capacidad de carga social y ambiental de la zona. Sin embargo, para sentar las bases del concepto y para su mayor comprensión se hace, acto seguido, un recorrido genérico de todas sus fases de desarrollo, tal y como las expuso su autor, Richard W. Butler.

\section{LAS ETAPAS DEL CICLO DE VIDA DE UN ÁREA TURÍSTICA DE RICHARD W. BUTLER}

\subsection{ETAPA DE EXPLORACIÓN}

En esta primera etapa, se encuentran los turistas más aventureros. No viajan bajo el auspicio de los grandes operadores de tours, ni aún con agencias de viajes. Hacen sus propios planes para sus viajes y siguen patrones de visita bastantes irregulares. Se puede esperar que estos turistas provengan de lugares más bien lejanos y que se sientan atraídos al destino por sus cualidades naturales y culturales únicas y diferentes. En esta etapa no se encontrará en el lugar ningún tipo de estructuración que se hubiese desarrollado a favor de la industria turística.

Por lo tanto, los servicios locales serán los que están a disposición de esta incipiente población turística y, en este momento, también se estima que el contacto con los residentes autóctonos será elevado. Este primitivismo estructural, al contrario de ser un menoscabo para este mercado, es un incentivo que satisface el apetito y los gustos de estos turistas. El tejido físico y el medio social de la zona permanecen relativamente inalterados por este tipo de turismo y, por lo tanto, representa muy poco a nivel social y económico para la vida de los residentes permanentes.

Esta etapa no es aplicable, en la actualidad, a la Costa del Sol ni al Mediterráneo, en general. Estos destinos se encuentran, en el presente, en la fase que algunos científicos han añadido como etapa adicional al CVAT. Esta se considera como la fase de madurez. Para cualificar como destino maduro, hace falta disponer de datos e información cuantificables y cualificables desde hace como mínimo 40 o 50 años. Aunque, históricamente, sí han pasado por esta fase de exploración. Concretamente, la Costa del Sol pasó por esta etapa en la última mitad del siglo XIX. Sin embargo, se encuentran, actualmente, en América Latina y en el Ártico canadiense, áreas por las que se sienten atraídos los turistas, sobre todo, por factores natrales y de índole histórico-culturales. 


\subsection{ETAPA DE PARTICIPACIÓN}

Siguiendo con el modelo de Butler, se continua con el proceso de evolución del destino turístico, y se pasa a la etapa de "participación" donde algunos de los residentes locales comienzan a suministrar servicios primordialmente, o hasta exclusivos, para los turistas. En este contexto, como en la anterior etapa, el contacto entre residentes y turistas es elevado y, hasta puede incrementarse para los residentes que suministran bienes y servicios para esta población invasora. Durante esta transición se puede esperar una incipiente campaña publicitaria dirigida a concienciar al mercado turístico para con esta localidad.

Según Butler, también se puede definir un mercado de área básico e inicial para los visitantes. Aquí, a la vez, se nota que emerge una temporada determinada de turismo y se tendrán que hacer reajustes en los patrones de actividad social, por lo menos entre la población nacional involucrada en esta industria. Ya se vislumbra alguna modalidad de organización y una estructura de viajes más sistematizados, y ya, los organismos oficiales responsables de estas zonas, se encuentran ante las primeras necesidades de montar o mejorar redes de transporte $\mathrm{u}$ otros servicios para los viajeros. Este patrón se exhibe, mayormente, en las islas del Pacífico o del Caribe y menos, aunque también, se ha comprobado que existen, en algunos lugares remotos de la Europa Occidental y América del Norte. España pasó por esta etapa históricamente, después de la Guerra Civil y antes del "boom" turístico de los años '60.

\subsection{ETAPA DE DESARROLLO}

Para Butler, cuando se inicia la etapa de "desarrollo", se observa un mercado turístico ya bien definido, gracias, en gran parte, a una campaña publicitaria formulada y dirigida a áreas que generan turismo. Con la evolución de esta etapa, la participación autóctona y el control local disminuirán notablemente. Esto se apreciará especialmente en la industria hotelera donde los establecimientos pequeños, en muchos casos administrados por familias locales, desaparecerán y se suplantarán por, a veces, cadenas hoteleras extranjeras más modernas y, quizás, sin tanta solera, pero con los adelantos que se ajustan al gusto cada vez más sofisticado de esta población aún más agrandada.

En esta etapa también se realizará una campaña de marketing específica dirigida a ciertas atracciones culturales y naturales. En algunos casos, se sustituyen estos últimos, por productos importados o artificiales, como ha sucedido con los campos de golf en el Mediterráneo español. Como símbolo de esta era, en Torremolinos, fue la construcción del Hotel Pez Espada, hotel de cinco estrellas. Bajo esta realidad habrá transformaciones en el espacio físico del lugar y estos cambios ya no contarán necesariamente con la capacidad decisoria de la población nativa.

Se hará necesaria la intervención pública a nivel provincial, regional y estatal como ha prevalecido en la Costa del Sol española, empezando por la creación del Ministerio de Información y Turismo y después, con el Patronato Provincial de Turismo de Málaga, a nivel provincial. Los residentes locales se pueden sentir desbordados por la población turística, al ser ésta más numerosa que aquélla durante las 
temporadas altas. Así, las novedades que se viven en el espacio pueden crear conflictos y desacuerdos entre ellos. Como cambio comercial, se encontrarán establecimientos jamás utilizados en estas sociedades, antes de esta transición, como las lavanderías y pizzerías. También, surgirá la necesidad de inmigración de otros lugares para cubrir los puestos de trabajo que se van creando para atender las crecientes necesidades del vertiginoso crecimiento de esta industria.

En este sentido, cabe mencionar al Arroyo de la Miel, barriada de Benalmádena que se destacaba por sus viviendas de alta densidad y de bajo coste, dedicadas al alojamiento de la mano de obra española, empleada en la construcción o la industria turística, en general. Este vecindario acabó por convertirse en el casco central del municipio de esta localidad costera. Sin embargo, su destino final fue su transformación en el barrio predilecto de los residentes británicos de bajos recursos económicos y de avanzada edad.

Además, como refugio residencial de los seres humanos que representan la fuerza bruta movilizadora de los pistones generadores energéticos del motor turístico de la región, está San Pedro de Alcántara, un pueblo localizado al oeste del casco urbano de Marbella. Esta localidad es la ciudad dormitorio para los empleados que trabajan en la construcción o en la industria del turismo, en general. Aquí, a diferencia de Arroya de la Miel, sus habitantes provienen de otras comarcas españolas, además del norte de África o de países europeos, como Rumania pero, también, del Lejano Oriente. Estos sectores componen dos tercios de la población activa ${ }^{3}$.

Este municipio merece mención, no sólo por ser el lugar de residencia de esta representación demográfica, sino también, por su cruda diferenciación económica, en un espacio geográfico tan reducido. Se pone en clarísimo contraste la presencia de los yates de los privilegiados de Puerto Banús, con respecto a la de los pisos pobres de los desposeídos, existentes en este pueblo.

\subsection{ETAPA DE CONSOLIDACIÓN}

Al acercase el momento de la consolidación, se puede afirmar que se ha logrado la fase más perseguida de todas, a pesar de que la tasa de crecimiento de visitantes se reduce. Este hecho numérico se puede pasar por alto, en este momento álgido del desarrollo de la industria, ya que, las cantidades absolutas de turistas todavía se incrementan, y el número de visitantes, en su totalidad, excede al número correspondiente de residentes locales. Además, aquí, la mayor parte de la economía de la zona tiene lazos con la industria del turismo. Se fortalecen las estrategias de marketing con el fin de extender la temporada del turismo y de maximizar el conocimiento del lugar a través de extensas campañas publicitarias. Se sigue con las grandes cadenas y franquicias de la industria pero hay pocas adiciones en este sentido.

\footnotetext{
3 Marchana Gómez, M. (1998). "Los impactos particulares del turismo en Andalucía: la configuación especial y demográfico-social de San Pedro de Alcántara”, Estudios Regionales, No 20, p. 102
} 
La influencia de tantos visitantes y tantos servicios que se prevé para ellos va a ser, inevitablemente, la causa de bastante descontento y oposición entre los residentes locales. Esta inconformidad se da especialmente entre los nacionales que no dependen, ni participan, ni se benefician, de las ganancias de esta industria, sobre todo si sus actividades y su "modus vivendi" se encuentra limitado por esta presencia importada. Esta tendencia se deja ver en zonas del Caribe o en la parte norte costera del Mediterráneo. Las ciudades turísticas de estas áreas tienen distritos comerciales para el ocio muy bien delimitados. Los no tan modernos se consideran como de segunda categoría y ya no tan deseables. En esta situación, el Patronato Provincial de Turismo de Málaga desarrolló el lema, “¡Al turismo una sonrisa!”, en reconocimiento a la actitud negativa que se hacía sentir por parte de los residentes hacia la población invasora.

\subsection{ETAPA DEL ESTANCAMIENTO}

Siguiendo con lo establecido por Richard Butler al pasar el área a la etapa del estancamiento, se llega al número máximo de visitantes. Se alcanza o se exceden los topes de la capacidad de carga para muchas variables con los problemas ambientales, sociales y económicos que conllevan tal situación. El área tiene una imagen bien establecida pero ya no está tan de moda.

Se confía en visitas repetidas y en congresos y convenciones para mantener el nivel de ingreso conseguido en la última etapa. Hay un exceso de oferta de camas disponibles y se necesita contar con mayores esfuerzos para mantener un nivel de entrada de turistas comparable a la etapa de consolidación.

Los atractivos genuinos y naturales se suplantan por servicios importados. La imagen del destino turístico se separa de su ambiente geográfico. Hay nuevo desarrollo que está en la periferia de donde existía la explotación turística original y las propiedades que existen, experimentan cambios de propietarios con frecuencia.

En este estudio, concerniente a la Costa del Sol, se llega a la conclusión de que se puede fijar este destino, en una nueva fase, creada por y para esta región turística, dada su condición de revestir semejanzas de dos estadios. Por un lado, este espacio se parece a la fase de la "consolidación", ya que a pesar de ser un destino maduro, posee la condición de seguir satisfaciendo los gustos del público viajero. En otras palabras, está todavía de moda. Este hecho se afirma, ya que, no se registra exceso notable de camas en temporadas estivales.

Por otro lado, también se registran similitudes con la fase siguiente, encontrándose, de esta manera, en el umbral del "estancamiento". Esto sucede, ya que, en tal estadio, los atractivos genuinos y naturales del lugar se han suplantado por servicios importados. En la Costa del Sol, estos servicios importados son, entre otras cosas, los campos de golf. Estas instalaciones se desarrollan en la periferia de donde previamente estaba, y sigue estando, la actividad turística principal, es decir, los cascos urbanos costeros.

Como en la fase del "estancamiento", estos campos deportivos han alterado la forma de la tenencia de la tierra, ya que, en su mayoría, existían, históricamente, cor- 
tijos donde se cultivaban patatas, pimientos y otros productos agrícolas. Es posible calificar a estas instalaciones deportivas como rasgos artificiales, ajenos al entorno geográfico andaluz, ya que el golf es un deporte importado y el diseño de sus estructuras no está en función de su espacio natural. Sus apariencias son iguales en todas partes del mundo.

También, la Costa del Sol está a punto de agotar sus recursos como el agua, en gran parte, por la demanda que los campos de golf hacen de este bien. El consumo desmesurado de este recurso tan apreciado, dentro del marco de esta España seca, está excediendo su capacidad de acogida ambiental. Como consecuencia inevitable de este estrés, también se le acompaña con problemas de la capacidad de acogida antropológica, ya que los residentes locales resienten los campos que no redundan ni económico ni ambientalmente en el bien de la mayoría. Así, por el cúmulo de estos factores, se puede colocar al destino, en la fase del "estancamiento", por un lado y, por otro, en la de la "consolidación".

De esta manera, se establece un nuevo estadio dentro del CVAT, definido por las características propias de este espacio. Por estos determinantes, socioeconómicos y ambientales, se crea la necesidad de formular otra categoría dentro del CVAT, ya que esta zona se sitúa "a priori" en la fase del "estancamiento" y "a posteriori" en el estadio de la "consolidación", denominándose así, el del "pre-estancamiento" Además, como ningún aparato teórico se ajusta plenamente a la realidad, cabe la adecuación de las características propiamente dichas del CVAT, a esta nueva adición, concebida a la medida de la realidad Costa Soleña.

\subsection{ETAPA DEL DECLIVE}

Siguiendo con la ortodoxia de Butler, la siguiente fase es la de declive. En esta etapa, el área no podrá competir con localidades nuevas y así menguará tanto a nivel espacial como en el número de turistas. Ya no tendrá ese atractivo para un turismo de tipo extendido sino que atraerá a visitantes más bien de temporada reducida, ya sea para un fin de semana o inclusive para estancias de un solo día si se tiene acceso para grupos numerosos de personas. Se puede notar también que la propiedades que estaban dispuestas para finalidades de turismo ahora empiezan a convertirse a usos no relacionados con este fin. Algunos científicos afirman que el momento de la caída se aproxima cuando se acorta la longevidad de las visitas. Sin embargo, no se sabe, como en el caso de la gallina y el huevo, si la decadencia causa el acortamiento de las visitas o viceversa.

En este punto, la participación de la población residente del lugar, en la industria del turismo, se incrementa como sucede en las primeras dos etapas de este ciclo. Este fenómeno se da porque el precio de la propiedad de los bienes inmuebles decae dada la misma dirección que sufre la acogida del destino turístico por los visitantes. De esta manera, se hacen más asequibles estos bienes para la población nativa. 


\subsection{ETAPA DE REJUVENECIMIENTO}

De acuerdo con Butler, el destino del desarrollo de la zona turística todavía tiene la capacidad de salvarse si en vez de entrar a la etapa de decaimiento pasa a la de rejuvenecimiento. Hay que notar que este cambio de dirección sólo se puede llevar a cabo, según este científico, si existe un cambio completo de la identidad en la que se basa la atracción turística. Esto se puede realizar por estímulos del sector público o privado.

Existen ejemplos marcados de este tipo de rejuvenecimiento. Primero está el de Atlantic City. Esta ciudad se salvó del ocaso por la adición y construcción de los casinos para complementar y reavivar el ya decaído ambiente de turismo de playa veraniego. Con estos casinos se prolonga la etapa turística cuantitativa y cualitativamente. Es decir, el turismo ha crecido numéricamente, y las visitas de estos grupos se da a lo largo de todo el año. Claro está, que este efecto sólo se realizará si no existen lugares cercanos que compiten con ofertas de producto semejantes. El éxito de Atlantic City se puede atribuir a su carácter único en esta dimensión regional costera del noreste de los Estados Unidos.

También se puede citar a Europa, en particular, con la instauración de balnearios y casinos que causan el mismo efecto en el destino que aquéllos en Atlantic City. Aunque el juego y el disfrute de estos centros de descanso y de tratamientos, con manantiales propios, han existido, desde hace mucho, en este continente, en la actualidad, gozan de mayor auge.

En la Costa del Sol española, existen dos casinos y como mínimo, aunque es todavía pequeña su oferta y demanda, media docena de clínicas y hoteles que ofrecen servicios variados como hidroterapias, tratamiento de algas, talasoterapias y revitalizaciones que están funcionando durante todo el año. Desafortunadamente, en la costa, propiamente dicha, no existen balnearios naturales. Estos están localizados en el interior en Carratraca y Tolox y se encuentran un poco fuera del área turística circundante.

Sin embargo, para mantenerse dentro de la deseada fase de la "consolidación" o bien para tomar nueva vida en el estadio del "renacimiento", un destino turístico, y en este caso particular, la Costa del Sol española, ha de apostar, de acuerdo con Butler, por un atractivo que le prolongue su temporada turística. Esta estrategia se ha realizado, con éxito, en un sentido de turismo cualitativo y cuantitativo, mediante el desarrollo de sus numerosos campos de golf. Dado su clima invernal benigno, las estaciones de primavera, otoño e, inclusive el invierno, son las temporadas altas para practicar el golf, ya que, es la época cuando, difícilmente, pueden los aficionados británicos y alemanes, dedicarse a este entretenimiento en sus países nativos. Los jugadores de estas nacionalidades componen, principalmente, el elemento demográfico que juega este deporte. Se completa el ciclo anual de la puesta en práctica del golf, con el verano clásico, cuando los turistas disfrutan además del mar, sol y playa. 


\section{LA ESCASEZ DEL RECURSO HÍDRICO Y SU NECESARIO USO DESDE EL DESARROLLO SOSTENIBLE}

Así, con la introducción del golf en su espacio, La Costa del Sol ha conseguido un turismo de mayor cantidad y calidad. Sin embargo, como se ha dicho antes, esto se ha desarrollado sin contar con las medidas preventivas para resguardar los recurso acuíferos de la región. Hasta se ha llegado al punto de que no da abasto la coexistencia del suministro de agua para las necesidades del consumo de la población conjuntamente con el de los campos de golf, poniendo en peligro la vida diaria de los turistas de playa y la de los ciudadanos del lugar.

Además la vegetación foránea que aparece en estos campos, y todos los aditivos químicos que esta requiere pone aún más hincapié en su antinaturalidad, con respecto a su entorno. No obstante, la característica artificial más destacable es su consumo de agua. Su apetito insaciable de este recurso agotable no ajusta a lo que ofrece el clima de la Costa del Sol, al pertenecer esta a la "Iberia Seca".

Alejandro López López tiene que decir lo siguiente acerca del elemento de la sequedad de la Península Ibérica y expresa con mayor claridad la presión de la capacidad de carga ambiental que ejercen los campos de golf en la zona:

“...el carácter 'seco' no es tanto consecuencia de unas precipitaciones escasas -que también en algunas zonas-, sino que éstas son irregulares, tormentosas y con una alta evapo-transpiración, presentando un 'balance hidro natural' deficitario a lo largo de buena parte del año, con rasgos marcadamente mediterráneos. Este se ha compensado a través del uso de agua subterránea, $\mathrm{y}$, mediante la selección de plantas y especies animales que se adaptaban a este tipo de necesidades. Además, esto se ha visto complementado por dos hechos recientes la expansión imparable del sector turístico residencial, y, de la agricultura hortofrutícola." 4 .

Por lo tanto, se puede analizar la sostenibilidad de la Costa del Sol si se le aplica el CVAT al destino, ya que, como sostienen Martin y Uysal, en un artículo suyo que es clave para el entendimiento de la relación entre la sostenibilidad y el CVAT:

"It is impossible to determine tourism carrying caacity outside of the context of the position of the destination area in the life cycle. The interrelationship of the two concepts is dynamic, with the idea of change implicit in both concepts. The carrying capacity for a destination area is different for each lifecycle"5.

De ahí, se puede afirmar que la Costa del Sol está en la etapa del "pre-estancamiento", ya que entre otras cosas ha excedido su capacidad de acogida ambiental por

${ }^{4}$ López López, A. Invitación a la educación ambiental: Recursos hidricos, desarrollo sostenible y gestión compartida, Asosiación Española de Educación Ambiental, Madrid, p.22.

5 Martin, B.S. y Uysal M., "An examination of the Relationship Between Carrying Capacity and the Tourism Life Cycle: Management and Policy Implications", Journal of Environmental Management and Policy Implications, 31, (1990) pp.320. 
su consumo, por parte de los campos de golf, del agua. Esto, a su vez, ha desencadenado la reacción de la superación de la carga antropológica, al existir un rechazo, por parte de la población local, de estas instalaciones turísticas, por su agotamiento de los recursos acuíferos de la localidad. Sin embargo, hace falta seguir haciendo hincapié, en el hecho de que es un destino que no ha dejado de estar de moda.

Se puede concluir, dada la fase del CVAT, en la que actualmente se encuentra la Costa del Sol, que no es un destino sostenible, por haber excedido las últimas dos capacidades de carga mencionadas. Sin embargo, sí puede, todavía, alcanzar el estadio tan deseado del "renacimiento" que sería la etapa que corresponde a la sostenibilidad. Inclusive, habría que afirmar, que ya tiene la mitad del camino recorrido, al haber construido los campos de golf que prolongan su temporada de turismo.

Para ganar la batalla al enemigo de la insostenibilidad solamente ha de convertirse el regadío de estas instalaciones deportivas a agua reciclada, sustituyendo, así, el actual uso que hace, en su mayoría, de agua de pozos. Siguiendo esta política de reciclaje acuífero, los campos de golf existentes y los que están por existir, pasarían a ocupar un puesto en "pro" de la comunidad Costa Soleña, tanto a nivel económico como ambiental, convirtiéndose en una fuerza democrática que beneficia a la sociedad entera.

Para tratar el tema del ecosistema, científicamente, es necesario también, abordarlo poéticamente, definiéndose, así, uno de sus compuestos principales. Esto lo consigue, Alejandro López López, en esta cita suya, al saludar y realzar elocuente y poéticamente la importancia del agua, ingrediente tan vital para el universo, y la vida terrenal.

López López dice lo siguiente al respecto:

"El conocimiento humano no concibe la existencia de vida sin la resencia de agua. La identificación de moléculas de agua es celebrada con alborozo por los astrónomos, como principal rastro de posibles vidas externas a nuestro Planeta. La molécula de agua, sencilla en su composición y estructura, reúne sin embargo unas propiedades que la hacen excepcional: capacidad de disolución de numerosas sustancias, estabilidad, alta inercia térmica y, lo que es más importante, se mantiene en estado líquido dentro del rango de temperaturas que se asocia con la vida. El Libro del Génesis saluda la importancia capital del agua, estableciendo su creación justo por detrás de la Luz y anterior a aquella de la flora, fauna, estrellas y, por supuesto, a la propia especie humana ${ }^{6}$.

\section{CONCLUSIÓN}

Para concluir, la eficaz gestión del agua es necesidad primordial para la existencia futura de la Costa del Sol, como ente turística sostenible pero, además, para su presente como ecosistema Mediterráneo. Si los campos de golf siguen consumiendo, para su riego, el agua de pozo, en vez del líquido reciclado, se pondrá en entredicho

${ }^{6}$ López López, A. Ibid. pp. 13 
la sobrevivencia misma de la región, como hábitat, de la vida vegetal y animal, a nivel más básico y primordial.

\section{BIBLIOGRAFÍA}

ARENAS GÓMEZ, A; MAJADA NEILA, J. (2003). Viajeros y turistas en La Costa del Sol, de Rilke a Brenan. Málaga: Editorial Miramar.

ARGAWAL, S. (2006). "Coastal Resort Resturcturing and the TALC", Richard W. Butler (Ed.). The Tourism Area Life Cycle. Conceptual and Theoretical Issues. Vol.2, pág. 201-230. Channel View Publications, Clevedon.

AMBIENTUM (2004 Enero). "Riego de campos de golf". Revista Ambientum.

BARKE, M; FRANCE, L. (1996). "The Costa del Sol". M. Barke \{et al\} (Ed.). Tourism In Spain: Critical Issues. Págs. 265-308. Cab International, Wallingford.

BEJARANO, J. (2005, 2 de agosto). "El monstruo insaciable de la Costa del Sol". La Vanguardia.

BORRELL MERLÍN, M. D.: "Turismo, Medio Ambiente, y Desarrollo Sostenible en el Mediterráneo". En Rev. OBSERVATORIO MEDIOAMBIENTAL. Vol. 8, Madrid, 2005, págs. 305-330.

BORRELL MERLÍN, M. D.: "Las políticas de Medio Ambiente en la UE y el litoral Mediterráneo español" en Rev. OBSERVATORIO MEDIOAMBIENTAL. Vol. 10, Madrid, 2007, págs. 325-337.

BUTLER, R.W. (1980). "The Concept of a Tourist Area Cycle of Evolution: Implications for Management of Resources". Canadian Geographer. Vol. 24.

Núm. 1, págs. 5-12.

BUTLER, R.W. (1997). "Modelling Tourism Development. Evolution, Growth and Decline". Tourism, Development and Growth. Wahat, S. y Piyram, J.J. (Eds.). Routledge, Londres, Nueva Yourk. págs. 109-122.

BUTLER, R.W. (ed.) (2006). Aspects of Tourism. The Tourism Area Life Cycle.

Vol. 2. Clevedon: Channel View Publications

DIPUTACIÓN DE MÁLAGA. (2007). El Turismo Residencial y de Segunda Residencia en la Provincia de Málaga. Málaga: Sociedad de Planificación y Desarrollo.

EL PAÍS (2006, 8 de agosto). "Campos sin urbanizaciones y regadíos". El País.

GRENON, M; BATISSE, M. (1989). Futures for the Mediterranean Basin: The Blue Plan. Oxford: Oxford University Press.

GUILLEN .F. (2005). The Rise of Spanish Multinationals: European Business in the Global Economy. Cambridge: Cambridge University Press.

LA CALA RESORT NEWSLETTER (2007). La Cala Resort Newsletter. Vol. 26, núm. 4, verano, 7.

LÓPEZ LÓPEZ, A; BORELL MERLIN, M.D. (1986) "Historia del derecho y de la política de las comunidades europeas en material de medio ambiente; perspectivas para España". Implicaciones constitucionales y políticas del ingreso de España e la C.E.E. y su incidencia en las comunidades autónomas; actas del V Congreso. Págs. 293-300 
LÓPEZ LÓPEZ. A. (1990) El Espacio Ambiental Europeo. Edición de la Universidad Complutense de Madrid y del Instituto Nacional de Consumo, 392 páginas. Madrid.

LÓPEZ LÓPEZ, A. (1990) La Comunidad Europea y la conservación de la naturaleza. Universidad Complutense de Madrid, ICONA. Madrid, 248 páginas.

LÓPEZ LÓPEZ, A. (1995) Introducción a la Sociología Ambiental y del Consumo.

$2^{\mathrm{a}}$ Edición, Instituto Nacional del Consumo. Madrid, 164 páginas.

LÓPEZ LÓPEZ, A. (2001). "Turismo y Desarrollo Sostenible”. Sistema. Núm. 162163, pág. 189-202. Junio 2001, Madrid.

LÓPEZ LÓPEZ, A. (2002). "La política medioambiental de la Unión Europea en materia de cambio climático". Observatorio Medioambiental . Vol. 5, págs. 163-191.

LÓPEZ LÓPEZ, A. y BORRELL MERLÍN, M. D. [Dirección y coordinación] (2005) Medio Ambiente y Desarrollo Sostenible en los países Mediterráneos de la Unión Europea. 393 páginas.

LÓPEZ LÓPEZ, A.; DE ESTEBAN CURIEL, J. (2008). “Turismo, Internet e indicadores ambientales de sostenibilidad". Observatorio Medioambiental. Vol. 11, págs. 185-199.

LÓPEZ LÓPEZ, A. (2011), Invitación a la Educación Ambiental: Recursos hídricos, Desarrollo Sostenible y Gestión compartida. Granada y Madrid: Asociación Española de Educación Ambiental. Madrid, 36 páginas.

MARCHENA GOMEZ, M. (1998). "Los impactos particulares del turismo en Andalucía: la configuración espacial u demográfico-social de San Pedro de Alcántara”. Estudios Regionales. Núm. 20, págs. 89-108.

MARTIN, B.S.; UYSAL, M. (1990). "An Examination of the Relationship Between Carrying Capacity and the Tourism Life Cycle: Management and Policy Implications". Journal of Environmental Management and Policy Implications. Vol. 31. Págs. 327-333.

MIRANDA PAEZ, E.; MUÑOZ LUQUE, P. (2007). Jornadas Internacionales de Golf y Medioambiente. 14 al 16 de marzo. Roquetas: ACOSOL S.A.

PACK, S. (2006). Tourism and Dictatorship: Europe's Peaceful Invasion of Franco's Spain. Nueva York: Palgrave Macmillan.

PÉREZ, P.L. (2011) El ciclo de vida de un área turística: aplicación a la Costa del $\mathrm{Sol}$. Tesis Doctoral leída en la Facultad de Ciencias Políticas y Sociología (UCM) el día 15 de julio, obteniendo la máxima calificación. 268 páginas y 15 de anexo.

SOTELO NAVALPOTRO, José Antonio. (Elaboración) La huella hídrica española en el contexto del cambio ambiental. Fundación Mapfre, Madrid, diciembre de 2010. Págs. 216.

SOTELO NAVALPOTRO, José Antonio, SOTELO PÉREZ, M; y GARCÍA QUIROGA, F: Análisis: "coste-beneficio" y "coste-eficiencia": la Huella Hídrica en España. Observatorio Medioambiental. Vol. 14, Madrid, 2011. 